\title{
Flowering Phenology in Selected Plant Communities of Grand Teton National PaRk
}

\author{
RON SCOGIN $\downarrow$ UNIVERSITY OF LA VERNE \\ LA VERNE $\uparrow \mathrm{CA}$
}

\section{$\downarrow \quad$ INTRODUCTION}

In the course of data collection for studies examining plant-animal species interactions within ecological communities, many various and diverse types of field data are collected. However, one such class of field data which are seldom systematically collected and reported is that of the natural flowering phenology of a particular plant community. These flowering phenology data describe the time sequence of flowering (initiation, duration, termination) for all of the plant species occurring in that community. The dearth of such data is suprising in light of its importance for the testing of models describing the factors influencing timing of plant reproduction events within communities and for investigating the possible evolutionary selection pressures which may have acted to modify plant phenologies. Limited sets of flowering phenology data have been presented for only a few scattered commnities, including tall-grass prairie (Anderson and Schelfhout, 1980); dry, tropical forest (Frankie, Baker, and Opler, 1974); spring woodlands (Schemske et al., 1978); Canadian marshes/bogs (Pojar, 1974); and subalpine meadow (Holway and Ward, 1965). These reports have consisted of limited data sets collected for narrowly explicit studies. The present report summarizes the results of a flowering phenology survey of selected communities within the Grand Teton National Park ecosystem for the first half of the flowering season (June - July) of 1996.

\section{MATERIALS AND METHODS}

The flowering status of species within each examined community was determined by direct observation while hiking a fixed path through each community at approximately 5-day intervals. The flowering status of each species was scored as: 1) first flowers noted, 2) early flowering, 3) peak flowering, 4) late flowering, and 5) last flowers noted. The following communities were examined:

(1) A riparian canyonside: Paintbrush canyon from Leigh Lake (6880') to the switchbacks at $8560^{\prime}$. This area represented both Conifer Forest and Subalpine Meadow,

(2) Conifer Forest/Sagebrush Scrub ecotone: the area surrounding the University of Wyoming/National Park Service Research Center (AMK Ranch),

(3) Sagebrush Scrub: Sagebrush flats along the road south of Signal Mountain (connecting with RKO Road).

\section{$\downarrow \quad$ RESULTS}

The results of the community flowering phenology surveys are summarized in Tables 1-3. For each date indicated, the flowering status of tabulated species is recorded as:

1-first flowers observed

2-early flowering 
3-peak flowering

4-late flowering

5-last flowers observed

\section{DISCUSSION}

Before the data presented in this report can be critically and usefully analyzed, the corresponding community flowering phenology data for the remainder (second half) of the flowering season (July-September) must be collected.

When compared with anecdotal flowering phenology reports for Grand Teton National Park (Craighead, 1994), the present data suggest that the initiation of the1996 flowering season was delayed by approximately 5 weeks. This was most likely due to an uncommonly high snowfall and late winter storms during the 1995-1996 season. However, by mid-June, the flowering phenology was consistent with reported dates (Craighead, 1994). This observation suggests that an extended winter has the effect of "telescoping" the early flowering season timing into a shorted interval, rather than shifting the entire season to later flowering dates. By mid season (mid-June), physiological mechanisms have "reset" flowering phenology back to a standard, "normal" schedule.

\section{LITERATURe CITED}

Anderson, R. C. and S. Schelfhout. 1980. Phenological patterns among tall grass prairie plants and their implications for pollinator competition. Amer. Midl. Nat. 104: 253-263.

Craighead, Frank, Jr. 1994. For Everything There Is a Season. Falcon Press, Helena, MT. $206 \mathrm{pp}$.

Frankie, G. W. , H. G. Baker, and P. A. Opler. 1974. Comparative phenological studies of trees in tropical wet and dry forest in the lowlands of Costa Rica. Jour. Ecol. 62: 881-919.

Holway, J. G. and R. T. Ward. 1965. Phenology of alpine plant in northern Colorado. Ecology 46: 73-83.

Pojar, J. 1974. Reproductive dynamics of four plant communities of southwestern British Columbia. Can. Jour. Bot. 52: 1819-1834.

Schemske, D. W., M. F. Willson, M. N. Melampy, L. J. Miller, L. Verner, K. M. Schemske, and L. B. Best. 1978. Flowering ecology of some spring woodland herbs. Ecology 59: 351-366. 
Twbie :

FLORAL PHENOLOCY: Conifer forealsagebrush scrub sotoses Location: Roscarch Cooveri AMK Reach

\section{Plear soxies}

Friolleris pudica (Purab) Spreag.

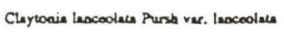

Violes awcelliene Punth

Viole purpurea Kell. var. veocoses (Woles.) Bria

Hydrophyllum ceppicaim Dougl. ex Beach.

Maboais repeses (Lindl.) G. Doa

Locicers ueshensis Wres.

Vaccinium scoperium Leib. ex Cov.

Amice cordifolis Hook

Wyechie beliancboidea Nure.

Collinesis parvifion Liodl.

Lomecium ambiguum (Nure) Coult. \& Rose

Lichoparayma giebrum Nuce

Belsemortizar segiterea (Pursb) Nutc.

Delphinium surtallienum Pricel ex Wuppen

Microsteris gracilis (Hook.) Greano var. Gracilis

Arabis bolboellii Horsece.

Frageris vesca $L$

Geranium viscoesium F. \& M.

Fritllaria atropurpure

Amoleachior alnu folis (Nuar.) Nutt. ex Roem.

Hackelia micrasche (Eessw.) Genury

Aodronace septeatrioaslis $L$ ver, subulifer

Lupinus argedous Punso.

Talespi arveases L. [Lacroduced]

Pocoocilis gracilis Dougl. ex Hook.

Trifolium loogipes a. Nels.

Agroecris glavea (Purna) Ref.

Ptacelia becorophylls Pursh

Crypleanthe Lorroyeas (Gray) Groece

Cborispors teoella (Pellea) DC.

Cescillejs linerrifolis Benth.

Sesecio integerrious Nute.

Galium sperino L

Eriophyllum lenerum Pursh

Aagelica pinneata Whas.

Linanchue sepponctionalis Mesoa

Ipomopsis aggregata (Pursa) Granc [Giliz aggregata]

Eriogooum umbollecum Tortey

Gayophytum diffueum T. \&O

Pensiemon cyeneochus Hook.

Erysimum cbeirantboides $L$

Cesochus velucious Dougl. ex Hook.

Collomis linearis Nure.

Valarienes odulis Nuce, ex T. \& C

Anemone multifida Poirot

Acbilles millefolium $L$ $\begin{array}{llllllll}\text { D.we: } 6 / 1 & 6 / 4 & 6 / 6 & 6 / 11 & 6 / 17 & 6 / 22 & 7 / 1 & 7 / 7\end{array}$

$3+4 . . . .$.

$3444 . .5$

. 4,5 . .

$\begin{array}{lllllll}2 & 2 & 3 & 4 & - & -\end{array}$

12344.

122345 .

$1245 \ldots$

123445 .

$134 . .$.

1345 .

$\begin{array}{llllll}1 & 2 & 2 & 4 & 5\end{array}$

14445 .

$134+45$

12445

$\begin{array}{llllll}1 & 2 & 4 & 5 & \end{array}$

12345 .

3445.

12344

$122+$.

$145 .$.

2345 ,

$\begin{array}{llll}1 & 2 & 3 & 4\end{array}$

12

1223

1234

4

234

123

123

123

25.

233

33

3

44

122

123

233

135

122

24

223

34

12

13 isote I (Coatinued)

Piner seriea

Duce: $6 / 1 \quad 6 / 4 \quad 6 / 6 \quad 6 / 11 \quad 6 / 17 \quad 6 / 22 \quad 7 / 1 \quad 7 / 7$

Suderis loagifolis Mual. ex Willd

Trepopogron dubiue Scop. (Introducal)

Carysencbermura laucanchemum L, (Introducal)

Hodieacbella uniflone (Nuar.) T. \& G.

Seoscio stroplendbifolius Groene

Spirea berulifolis Palles 
Twbie 2

FLORUL PHENOLOCY: Conifer WoOdland/Subulpine Meadow Location: Leigh Lake/Paincorush Cenyon Plsolsosiat

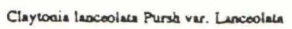

Loaicen ulebonsis Wase.

Kelmis micropbylls (Hook.) Heller

Vaccinium membranecoum Dougl. ex Torrey

Riber viscosicerimum Pursh

Valariane adulis Nura. ex T. \& G.

Hydrophyllum ceppicerum Dougl. ex Bench.

Fritilleris pudica (Pursh) Spreag.

Calypeso bulbosen (L) Oakes

Veccinium scoperium Leib. ex Cor.

Araica cordifolis Hook.

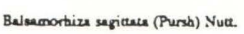

Arabis bolboellii Horsem.

Maboais repens (Liodl.) G. Dos

Menriesis ferruginea Smith

Castilleja angustifolis (Nuta.) G. Doo [Cestilleja chromose ]

Reaunculus eschacboiteris Sctiocebct.

Astennaria racemoes Hook.

Clemecis occideoulis (Horbem.) DC. var. grossesorrats (Rydb.) Pringle

Pedicularis bractioosen Benth.

Thelictrum fendler Eogelm ex Gray

Geranium viscosiscionum F. \&....

Fragaria vesca $L$

Erysimum cbeiranchoidea $L$.

Lomecium triveresenum (Pursh) Coult. \& Row

Actea rubra (Niwa) Willd.

Collinsis perviflon Lindl.

Viols autalliene Pursen

Asconneris dimorpas (Nuct.) T. \& G.
Table 3

FLORAL PHENOLOGY: Sagebrumb scrub

Locesion: Noer RKO Rowd, south of Signel Mountesin

Plantspociee

Dodecartheon conjugens Groene

Viola nuralliana Pursb

Viola purpuree Kell. ver, venava (Wale.) Brain

Ciayronia lanceolara Pursh var. Lancrolata

Fritillarie pudica (Purath) Spreng.

Lomarium ambiguwn (Nua.) Coult. \& Rose

Collinsia parmilora Lindl.

Lithophragma glabrum Nurt.

Delphiniwm nurallianum Prizel ox Walpers

Nrabis holboellii Horbeen.

Maianthemwm stellaswn (L.) Link [Smilacina stellaua ]

Senecio incegerrimus Nuck.

Castillega curidbi Groenm.

Fritillaria arropurpurea Nutc.

Lupinur sericew Pursh.

Purshia ridentata (Pursb) DC.

Collomia limearis Nure

Zigadenas paricularus (Nurt.) Wats.

Gewn sriflorum Pursh

Crepis arrabarba Heller

Erigeron glabellus Nuac.

Dale: $\quad 6 / 1 \quad 6 / 8 \quad 6 / 13 \quad 6 / 20 \quad 6 / 26$

$23 .$.

$23 .$.

$23 .$.

, $5 . .$.

, . .

1234

$2+4$.

, 5.

$13+4$

$1,4+4$

2225

234

12

+ .

12

12

13

14

13

24

Eriogonum umbellarum Torrey

Eriophyllum Lanarum Purno

Lower portion of rand- Eust of RKO Rowd junction

Balsamartiza sagiraua (Pursh) Nuck.

Mieraseriv grasilis (Hook.) Grocke ex Walpers

Lithorpormum ruderale Dougl. ex Lehm.

Fragaria verca $L$

Lamarium cour (Wace.) Coulc. \& Roses

Viola acunce Smich

Gnapalium palustre Nutr.

Maianshomum stellawum (L.) Link [Smilacina stellara ]

Ranunculus cymbalaria Pursh var. alpinus Hook.

Ipamopsis aggregara (Purab) Grant

Alliwm brenisylum wate.

Mrer ancidenvalis (Nurc) T. \& G.

Agaseris glawea (Pursa) Raf.

Anemone multifida Poired

Achillea millifolium L

Linanchus seprentrionalis Mesoo 